
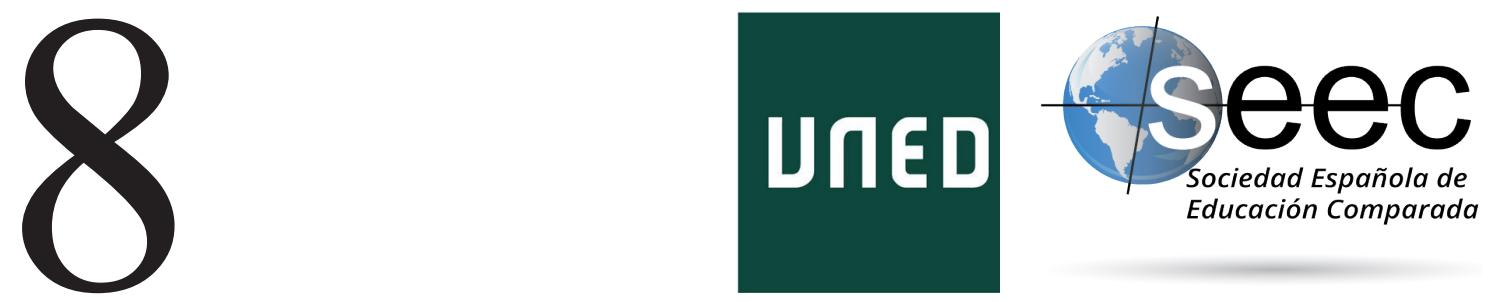

\title{
De la formación docente en España a la práctica educativa en Senegal: un proyecto de cooperación internacional basado en el aprendizaje servicio para Infantil y Primaria
}

From teacher training in Spain to educational practice in Senegal: An international cooperation project based on service learning for Pre-School and Primary Education

\section{Rocío López-García-Torres*; Elia Saneleuterio**; Nuria Andreu-Ato***}

DOI: $10.5944 /$ reec.39.2021.29393

\section{Recibido: 13 de Enero de 2021 Aceptado: 06 de mayo de 2021}

\footnotetext{
* Rocío López-García-Torres: Licenciada en Psicología y doctora en Ciencias de la Educación por la Universidad de Granada. Es profesora del Área de Psicología Evolutiva y de la Educación en el Departamento de Ciencias de la Educación de la Universidad CEU Cardenal Herrera (CEU Universities). Colaboradora del Grupo de Investigación TALIS y autora de diversos artículos publicados en revistas científicas, contribuciones a congresos de ámbito nacional e internacional y capítulos de libro.. Datos de contacto: E-mail: rocio.lopez@uchceu.es; ORCID: http://orcid.org/oooo-0001-5647-764X

** Elia SANELeuterio: Doctora Europea en Literatura Española, licenciada en Filología Hispánica, maestra en Educación Infantil y máster en Investigación en Didácticas Específicas. Es profesora de Magisterio en el Departament de Didàctica de la Llengua i la Literatura de la Universitat de València. Directora del Grupo de Investigación TALIS y autora de numerosos capítulos de libro, artículos de investigación y monografías.. Datos de contacto: E-mail: elia.saneleuterio@uv.es; ORCID: http://orcid.org/oooo-0003-4060-9518

*** NuRIa ANDREU-ATo: Licenciada en Pedagogía, experta en intervención logopédica y doctora por la Universidad CEU Cardenal Herrera (CEU Universities). Es profesora del Área de Psicología Evolutiva y de la Educación en el Departamento de Ciencias de la Educación de dicha universidad, en la que ha sido vicedecana de Educación en la sede de Elche. Es también autora de diversos artículos publicados en revistas científicas y contribuciones a congresos de ámbito nacional e internacional.. Datos de contacto: E-mail: nuria.andreu@uchceu.es; ORCID: http://orcid.org/oooo-0001-9808-545X
} 


\title{
Resumen
}

Se presenta y analiza un proyecto de cooperación internacional para alumnado universitario español, en el que se combina la metodología formativa de aprendizaje servicio (ApS) con una estancia en un país emergente. Desde los grados de Educación Infantil y Educación Primaria de la Universidad CEU Cardenal Herrera, se planificó y ejecutó una intervención educativa en Djikesse (Senegal), concretamente en las escuelas de Educación Infantil (Guardería Antoni Gaudí) y de Educación Primaria (Escuela Primaria de Djikesse). Participaron nueve maestros senegaleses y un total de 290 estudiantes entre ambas etapas educativas; por su parte, desde España se involucraron dos profesoras y ocho estudiantes de la universidad mencionada. Estos últimos, futuros docentes, tuvieron la posibilidad de completar su formación con esta experiencia. Los resultados evidencian diversos beneficios del proyecto, tanto para la comunidad educativa visitante como para la de acogida. Respecto a la parte de servicio o repercusión en Senegal, destaca la promoción de metodologías activas de aprendizaje, haciendo consciente al profesorado de la importancia de la experimentación y la observación, así como de promover un aprendizaje globalizador y significativo, además del avance en la concepción del juego y su papel en el aprendizaje. Como debilidad, pueden mencionarse las dificultades con los procesos cooperativos, debido a la falta de espacio y de recursos. En cuanto al aprendizaje, tras la autoevaluación del proyecto por parte de los participantes universitarios se concluye que habría sido necesaria una mayor implicación del alumnado en la fase de planificación, y especialmente en lo relativo a la evaluación. Con todo, la experiencia ha conseguido vincular la teoría con la práctica y sus beneficios son considerables, entre otros, la conciencia sobre el valor fundamental de la educación como instrumento para combatir la pobreza y la exclusión social.

Palabras clave: Magisterio; proyecto; cooperación internacional; práctica pedagógica; aprendizaje

\begin{abstract}
An international cooperation project for Spanish university students is presented and analyzed, in which the formative methodology of service learning (SL) is combined with a stay in an emerging country. From the degrees of Early Childhood Education and Primary Education of the CEU Cardenal Herrera University, a pedagogical intervention was planned and executed in Djikesse (Senegal), specifically in the Antoni Gaudí Nursery and the Djikesse Primary School. Nine Senegalese teachers and their 290 students participated between both educational stages. For their part, from Spain, two professors and eight students from the aforementioned university were involved. These future teachers had the opportunity to complete their training with this experience. The results show various benefits through the project, both for the visiting and for the host educational communities. Regarding the service part or impact on Senegal, highlights the promotion of active learning methodologies, making teachers aware of the importance of experimentation and observation, as well as promoting global and meaningful learning, in addition to advance in their conception of the game and its role in learning. As a weakness, the difficulties with cooperative processes, due to lack of space and resources, can be mentioned. Regarding learning, after the project self-evaluation by the university participants, it is concluded that a greater involvement of the students in the planning phase, and especially in the evaluation aspects, would have been necessary. However, this experience has managed to link theory with practice and its benefits are considerable, among others, awareness of the fundamental value of education as an instrument to combat poverty and social exclusion.
\end{abstract}

Keywords: Teaching; project; international cooperation; pedagogical practice; learning 


\section{Introducción}

El aprendizaje servicio (en inglés Service-Learning, y también conocido por sus siglas no normativas en español ApS) es una metodología educativa que combina el aprendizaje con el servicio a la comunidad. Mientras que el servicio puede ser de muy variada naturaleza (técnico, físico, intelectual...), el aprendizaje siempre es multidimensional: el desarrollo conceptual se ve enriquecido, principalmente, por el aprendizaje de habilidades y valores, además de aumentar la motivación dado el necesario carácter participativo de todo el proceso (Folgueiras et al., 2019; Lear y Abbott, 2019; Martín García et al., 2010). Así pues, el aprendizaje servicio se puede definir como «actividad o programa de servicio solidario protagonizado por los estudiantes, orientado a atender eficazmente las necesidades de una comunidad, y planificada de forma integrada con los contenidos curriculares con el objetivo de optimizar los aprendizajes» (Tapia, 2008, p. 43).

De manera intuitiva, esta metodología se aplica desde hace mucho tiempo en contextos diversos, principalmente en la educación no formal, a través de experiencias de servicio gratuito organizadas para adquirir conocimientos, pero que a la vez cubren necesidades sociales. Sin embargo, en las últimas décadas se ha realizado un esfuerzo de sistematización y categorización del aprendizaje servicio, primero en América y luego en Europa, aparte de que su propia práctica se ha multiplicado en la educación reglada. La metodología se ha revelado como exitosa, tanto si se analiza el aprendizaje generado (Rubio i Serrano, 2009) como si se analiza el servicio prestado (Battle, 2009), además de otra realidad que con ambos se posibilita: el aprendizaje comunitario (Lear y Abbott, 2019), así como el «fortalecimiento de un compromiso ciudadano con la Comunidad» (Rodríguez Gallego, 2014, p. 95). Entre sus beneficios, el ApS favorece la implicación del alumnado, por identificarse como un proceso de aprendizaje muy participativo (Folgueiras et al., 2019).

Rodríguez Gallego (2014) insiste en la importancia de diferenciar esta propuesta pedagógica de experiencias de aprendizaje práctico no solidario, y de iniciativas que sí son solidarias, pero que no tienen entre sus objetivos la formación profesional de quien ofrece el servicio. La tabla 1 categoriza las diferencias entre estas experiencias de voluntariado y el aprendizaje servicio.

Tabla 1.

Diferencias entre aprendizaje servicio y voluntariado y servicio comunitario

\begin{tabular}{lll}
\hline & Aprendizaje servicio & Voluntariadoy servicio comunitario \\
\hline Enfoque & $\begin{array}{l}\text { Enfoque pedagógico-solidario y met- } \\
\text { odología de enseñanza-aprendizaje }\end{array}$ & Enfoque pedagógico-solidario \\
\hline Objetivos & Servicio y aprendizaje & Servicio \\
\hline Formación & Profesional y ciudadana & Exclusivamente ciudadana \\
\hline Fases & $\begin{array}{l}\text { Requiere fases de preparación, acción } \\
\text { y reflexión }\end{array}$ & Comprende la actividad misma \\
\hline Normas & $\begin{array}{l}\text { Existen normas establecidas para el } \\
\text { desempeño del servicio }\end{array}$ & No existen normas establecidas \\
\hline $\begin{array}{l}\text { Planifi- } \\
\text { cación }\end{array}$ & $\begin{array}{l}\text { Requiere planteamiento anticipado a a } \\
\text { la fecha del servicio }\end{array}$ & Puede ocurrir en cualquier momento \\
\hline Supervisión & $\begin{array}{l}\text { Debe realizarse con guía o super- } \\
\text { visión adecuadas }\end{array}$ & Puede realizarse sin supervisión \\
\hline Certificación & Se acredita el servicio & No es un requisito \\
\hline
\end{tabular}

Fuente: adaptado de Rodríguez Gallego (2014, p. 97). 
Si enfocamos los intereses de la educación para la justicia global, cabe mencionar el potencial de las experiencias de aprendizaje desarrolladas en todo el mundo, especialmente en países emergentes (Monzó et al., 2018; Uruñuela, 2018). Tampoco podemos obviar que dentro de este ámbito educativo tienen una mayor relevancia las iniciativas que se enfocan en la propia educación, pues se ha remarcado desde siempre la importancia de la formación docente en la cooperación internacional (JRS, 2019), así como los beneficios del aprendizaje basado en el trabajo y la consecuente ampliación de los esquemas de formación (Psifidou y Ranieri, 2020). El estudio de Psifidou y Ranieri (2020) permite analizar el impacto de los programas de cooperación entre países. En este ámbito, la aportación de las universidades es reconocida desde hace décadas (Huarcaya et al., 2005). Ahora bien, el proceso también ha demostrado su validez a la inversa, siendo, por ejemplo, los maestros y maestras indígenas en formación quienes participan en programas de movilidad internacional (Hernández, 2019).

Dentro de este contexto, no son pocas las universidades que apuestan por ofrecer a sus estudiantes este tipo de formación que, a su vez, supone una colaboración solidaria con otros países, especialmente desde la metodología del ApS, que registra una tendencia ascendente a su institucionalización (Álvarez Castillo et al., 2017). En concreto, la Universidad CEU Cardenal Herrera (UCH CEU) tiene un doble compromiso: formativo, por un lado, con la transmisión del saber académico y el desarrollo del conocimiento científico, y, por otro, solidario, un compromiso con la diversidad de realidades sociales en desventaja, con el objeto de favorecer su transformación y progreso. Las universidades asumen la tarea de formar profesionales responsables que puedan asumir los retos del mundo como ciudadanos activos. En este sentido, la vinculación de la formación con el ApS es una excelente oportunidad de adquirir competencias sociales y éticas para la práctica profesional, dado que los matices en medio de los que el alumnado aprende resultaría más difícil obtenerlos de otra manera.

\section{Contextualización, objetivos y metodología}

Dentro de las iniciativas solidarias de la UCH CEU, este proyecto se contextualiza en la Misión Senegal, país con una de las localizaciones del voluntariado internacional de la citada universidad.

Pues bien, con las certezas mencionadas en el apartado anterior, desde las titulaciones de Educación de la UCH CEU se presentó un proyecto de cooperación internacional y aprendizaje servicio que sumaba a la Misión Senegal, donde hasta ahora solo se había implantado un proyecto relacionado con la salud bucodental, otro de intervención educativa en las escuelas de Educación Infantil y de Educación Primaria de Djikesse (Bignona), el cual procurara enriquecer la formación y contribuir a la actualización de los maestros de estas escuelas, con una perspectiva continuista, al tiempo que sirviera para completar la formación curricular de los propios estudiantes de Magisterio de la UCH CEU. El proyecto fue aprobado y financiado por la Cátedra Solidaridad ${ }^{1}$ del Vicerrectorado de Estudiantes y Vida Universitaria de la UCH CEU; asimismo, diversos medios de comunicación recogieron y explicaron esta iniciativa solidaria de cooperación internacional (Actualidad CEU, 2020a; AlicanteHoy.es, 2020; Información, 2020a, 2020c; Onda Cero, 2020), dando visibilidad al proyecto educativo.

$1 \quad$ Coordinada por el profesor Santiago Celestino Pérez Jiménez. 
Los objetivos de esta aportación son dos: (O1) presentar una experiencia de aprendizaje servicio y de cooperación con Senegal, así como (O2) analizar la repercusión tanto para el país receptor (O2.1) como para los estudiantes españoles que participaron en el proceso de movilidad internacional (O2.2). Así hubo un doble beneficiario: la comunidad educativa senegalesa de dos escuelas y un grupo de docentes en formación de una universidad española. Ello que explica que el segundo objetivo general se desdoble en dos, lo que permite, metodológicamente, diferenciar de una manera más clara el proceso de atención analítica de cada uno.

Para el primer objetivo (O1), se describe detalladamente la experiencia en un primer apartado de resultados, limitándose el presente a identificar a los sujetos y entidades participantes.

El objetivo O2, como se ha dicho, se bifurca en dos focos de atención. Ambos se analizan partiendo de la observación participante, aportando datos concretos del impacto de la experiencia principalmente en las entidades educativas del país de acogida (O2.1), y además el O2.2 se analiza con un instrumento adicional. Para poder interpretar los datos, y teniendo en cuenta que la iniciativa cuenta con pretensión tanto solidaria como educativa, cabe explicitar los objetivos pedagógicos del proyecto (que no deben confundirse con los objetivos investigadores expuestos arriba). Lo que se pretendía al diseñar el proyecto era desarrollar acciones solidarias orientadas a colaborar eficazmente con unas limitaciones socioeducativas concretas. Más específicamente, y centrándonos en el grupo de docentes en formación, se pretendía conseguir los siguientes resultados de aprendizaje:

1. Conocimiento de otros entornos sociales y educativos y comparación práctica con el sistema educativo español, desembocando en un ejercicio de reflexión sobre el diseño de intervenciones o propuestas de mejora futuras (Información, 2020d).

2. Aprendizaje de la profesión docente mediante la práctica, una buena oportunidad de adquisición de competencias para la práctica profesional.

3. Implementación de metodologías activas y enseñanza a los maestros y maestras senegaleses a utilizar determinados materiales didácticos y/o a confeccionarlos, optimizando los procesos de enseñanza-aprendizaje e incidiendo en su realidad educativa concreta. Este objetivo es, realmente, el que da sentido a los otros dos, dado que se trata de un proyecto solidario.

Finalmente, y como se ha dicho, para la consecución del objetivo O2.2, a su regreso a España todos los participantes universitarios completaron una rúbrica específica para la autoevaluación de proyectos de aprendizaje servicio (Serrano et al., 2015), disponible en la tabla 2. Asimismo, elaboraron una memoria reflexiva, con apoyo de sus anotaciones durante la estancia (diario de sesiones), que además fue presentada en el $17 .{ }^{\circ}$ Congreso Internacional de Estudiantes de la UCH CEU. 
Tabla 2.

Rúbrica para la autoevaluación de proyectos de aprendizaje servicio

I

Ignoradas. Las necesidades no están programadas ni se prevén actividades para detectarlas o definirlas, aunque es probable que estén presentes en el proyecto

II

III

IV

Presentadas. Los educadores y/o entidades sociales deciden las necesidades sobre las que realizar el servicio sin consultar a los participantes
Decididas. Los participantes, a menudo junto con los educadores, deciden las necesidades sobre las que quieren actuar, a través del análisis de diferentes problemáticas y la elección de una de ellas
Simple. Servicio de corta duración compuesto por tareas sencillas cuya realización supone una exigencia e implicación limitadas

\section{Tangencial. Servicio} que no parte de una necesidad detectada y del que los participantes no perciben su posible dimensión social

\section{Continuado. Servicio de duración prolon- gada compuesto por tareas repetitivas y/o fáciles de apren- der, cuya realización supone una exigen- cia e implicación moderadas}

\section{Complejo. Servicio} de duración prolongada que permite adquirir experiencia y destreza en la realización de tareas de notable complejidad, cuya realización supone una exigencia e implicación elevadas

Necesario. Servicio que da respuesta a una necesidad de la comunidad, aunque los participantes no siempre logran percibir su dimensión social
Cívico. Servicio que da respuesta a una necesidad de la comunidad y de cuya dimensión social los participantes son conscientes
Creativo. Servicio de duración variable compuesto por tareas complejas que los propios participantes deben diseñar para resolver un problema que exige creatividad, lo que supone incluso una exigencia e implicación mayores

Transformador. Los participantes dan respuesta a una necesidad y son conscientes de su dimensión social, pero además perciben los límites de cualquier servicio que no considere la acción política

\begin{tabular}{|c|c|c|c|}
\hline $\begin{array}{l}\text { Espontáneo. Los } \\
\text { aprendizajes no } \\
\text { están programados } \\
\text { y tampoco existen } \\
\text { actividades pensadas } \\
\text { para facilitarlos; se } \\
\text { adquieren de modo } \\
\text { informal durante el } \\
\text { servicio }\end{array}$ & $\begin{array}{l}\text { Planificado. Los } \\
\text { aprendizajes se pro- } \\
\text { graman de acuerdo } \\
\text { con el currículum o } \\
\text { proyecto educativo } \\
\text { y se diseñan activi- } \\
\text { dades para adquirir- } \\
\text { los, sin contemplar } \\
\text { necesariamente su } \\
\text { relación con el ser- } \\
\text { vicio }\end{array}$ & $\begin{array}{l}\text { Útil. Los apren- } \\
\text { dizajes planificados } \\
\text { y las actividades for- } \\
\text { mativas tienen una } \\
\text { estrecha relación } \\
\text { con el servicio. Su } \\
\text { adquisición favorece } \\
\text { una mejor calidad de } \\
\text { la intervención }\end{array}$ & $\begin{array}{l}\text { Innovador. Los } \\
\text { aprendizajes se adqui- } \\
\text { eren a partir de una } \\
\text { actividad investigado- } \\
\text { ra, están relacionados } \\
\text { con el currículum o } \\
\text { proyecto educativo y } \\
\text { se vinculan directa- } \\
\text { mente con el servicio a } \\
\text { la comunidad }\end{array}$ \\
\hline
\end{tabular}




\section{Cerrada. Los partic- ipantes se limitan a realizar las tareas que previamente se han programado para el desarrollo de la ac- tividad, sin la posib- ilidad de introducir modificaciones a la} propuesta inicial

\section{Indeterminado. Pro-} cesos espontáneos de ayuda entre participantes que realizan una actividad individual de servicio

\section{Delimitada. Los par- Compartida. Los ticipantes realizan aportaciones pun- tuales requeridas por los educadores en distintos momen- tos del proceso

so \\ participantes com- parten con sus educadores la re- sponsabilidad en el diseño y desarrollo del conjunto de la actividad}

Colaborativo. Procesos basados en la contribución de los participantes a un proyecto colectivo que requiere unir tareas autónomas e independientes so de trabajo interdependiente entre participantes en un proyecto colectivo que requiere articular aportaciones complementarias
Liderada. Los participantes se convierten en promotores y responsables del proyecto de modo que intervienen en todas sus fases, decidiendo sobre los diferentes aspectos relevantes
Cooperativo. Proce- Expansivo. El trabajo para alcanzar un objetivo común

Continua. Además

Difusa. La actividad reflexiva no está prevista, ni se proponen tareas para impulsarla, aunque de modo natural puede pensarse y someterse a debate la propia experiencia

Casual. No hay actividades de reconocimiento previstas, aunque de manera espontánea los diferentes agentes que intervienen pueden agradecer y valorar la tarea realizada por los protagonistas
Puntual. La reflexión está programada y hay tareas previstas para facilitarla, aunque ocupa solo un tiempo limitado y separado del curso de las actividades del proyecto

Intencionado. Los educadores organizan actividades destinadas a reforzar positivamente el trabajo de los participantes y/o a celebrar la finalización del servicio

\section{de contar con mo-} mentos y tareas de reflexión, los participantes llevan a cabo ejercicios reflexivos durante la realización de todo el proyecto

\section{Recíproco. Los ben-} eficiarios del servicio y/o las entidades sociales, a menudo en colaboración con los educadores, llevan a cabo iniciativas para expresar su gratitud y celebrar el éxito del servicio

\section{colectivo va más allá} del grupo inicial de participantes e incorpora de forma activa a otros agentes externos, creando así redes de acción comunitaria

\section{Productiva. La re-} flexión, además de prevista y continuada, implica a los participantes en una actividad de síntesis o de creación que produce una nueva aportación a la comunidad

\section{Público. El recono-} cimiento a los participantes adquiere una dimensión pública, bien porque la actividad se ha dado a conocer a la ciudadanía, o bien porque la administración la agradece y difunde por su valor cívico

\begin{tabular}{ll}
\hline Informal. No existe & Intuitiva. Para \\
un plan de evaluación & evaluar, los educa- \\
establecido, aunque & dores se limitan a \\
los educadores, de & constatar, sin crite- \\
manera espontánea & rios ni indicadores \\
y puntual, pueden & definidos, el logro \\
evaluar y comunicar & de ciertos objetivos \\
su valoración a los & generales de apren- \\
participantes & $\begin{array}{l}\text { dizaje, que pueden } \\
\text { acreditarse }\end{array}$
\end{tabular}

Competencial. Los educadores aplican un plan de evaluación que define objetivos, criterios, indicadores y metodologías para mejorar el desarrollo competencial de los participantes y para acreditarlos
Conjunta. Los participantes, junto con los educadores, intervienen de manera activa en diferentes momentos del proceso de preparación y aplicación de un plan de evaluación competencia 


\begin{tabular}{|c|c|c|c|}
\hline $\begin{array}{l}\text { Unilateral. En el } \\
\text { proyecto participa } \\
\text { una sola organi- } \\
\text { zación, normalmente } \\
\text { educativa, bien } \\
\text { porque se accede di- } \\
\text { rectamente al espacio } \\
\text { de servicio, o bien } \\
\text { porque el receptor } \\
\text { forma parte de la } \\
\text { organización que lo } \\
\text { impulsa }\end{array}$ & $\begin{array}{l}\text { Dirigido. En el } \\
\text { proyecto participan, } \\
\text { al menos, dos orga- } \\
\text { nizaciones: la educa- } \\
\text { tiva que lo planifica } \\
\text { y lleva a cabo y la } \\
\text { entidad social que } \\
\text { se limita a ofrecer el } \\
\text { espacio de servicio }\end{array}$ & $\begin{array}{l}\text { Pactado. Al menos } \\
\text { dos organizaciones } \\
\text {-una educativa y } \\
\text { otra social-acuerdan } \\
\text { conjuntamente las } \\
\text { condiciones de apli- } \\
\text { cación de un proyec- } \\
\text { to de aprendizaje } \\
\text { servicio diseñado } \\
\text { exclusivamente por } \\
\text { una de ellas }\end{array}$ & $\begin{array}{l}\text { Integrado. Las or- } \\
\text { ganizaciones impli- } \\
\text { cadas en el proyecto } \\
\text { lo diseñan y aplican } \\
\text { conjuntamente, desde } \\
\text { el inicio hasta el final } \\
\text { del proceso }\end{array}$ \\
\hline $\begin{array}{l}\text { Incipiente. El apren- } \\
\text { dizaje servicio se } \\
\text { conoce a causa de un } \\
\text { proyecto que ya lleva } \\
\text { a cabo algún educa- } \\
\text { dor/a o debido a la } \\
\text { presentación de una } \\
\text { experiencia de otro } \\
\text { centro }\end{array}$ & $\begin{array}{l}\text { Aceptada. Los } \\
\text { proyectos de apren- } \\
\text { dizaje servicio de } \\
\text { iniciativa personal } \\
\text { tienen el recono- } \\
\text { cimiento del equipo } \\
\text { directivo del centro y } \\
\text { el respaldo de parte } \\
\text { del profesorado }\end{array}$ & $\begin{array}{l}\text { Integrada. } \text { El apren- } \\
\text { dizaje servicio está } \\
\text { presente en más de } \\
\text { un nivel educativo, } \\
\text { se vincula al currícu- } \\
\text { lum de diferentes } \\
\text { materias, tiene } \\
\text { implicaciones en la } \\
\text { metodología y en } \\
\text { la organización del } \\
\text { centro }\end{array}$ & $\begin{array}{l}\text { Identitaria. El apren- } \\
\text { dizaje servicio forma } \\
\text { parte de la cultura del } \\
\text { centro, consta en su } \\
\text { proyecto educativo y } \\
\text { el centro lo presenta } \\
\text { como un rasgo de su } \\
\text { identidad }\end{array}$ \\
\hline $\begin{array}{l}\text { Incipiente. El apren- } \\
\text { dizaje servicio se con- } \\
\text { oce porque ya se ha } \\
\text { participado en alguna } \\
\text { experiencia puntual } \\
\text { o debido a la present- } \\
\text { ación del proyecto de } \\
\text { otra entidad }\end{array}$ & $\begin{array}{l}\text { Aceptada. Los } \\
\text { proyectos de apren- } \\
\text { dizaje servicio tienen } \\
\text { el reconocimiento de } \\
\text { la dirección y de los } \\
\text { profesionales de la } \\
\text { entidad, que aceptan } \\
\text { la función educadora } \\
\text { de la organización }\end{array}$ & $\begin{array}{l}\text { Integrada. El apren- } \\
\text { dizaje servicio está } \\
\text { presente en el pro- } \\
\text { grama de actividades } \\
\text { de la entidad, que } \\
\text { cuenta con la estruc- } \\
\text { tura y el personal } \\
\text { necesarios para } \\
\text { asegurar su imple- } \\
\text { mentación }\end{array}$ & $\begin{array}{l}\text { Identitaria. El apren- } \\
\text { dizaje servicio forma } \\
\text { parte del ideario de la } \\
\text { entidad, que lo pre- } \\
\text { senta como un rasgo } \\
\text { propio de su identidad } \\
\text { y lo dota de los recur- } \\
\text { sos necesarios para } \\
\text { asegurar su imple- } \\
\text { mentación }\end{array}$ \\
\hline
\end{tabular}

Fuente: tomado de Serrano et al. (2015, pp. 120-121).

\subsection{Participantes y organismos implicados}

La planificación de la experiencia parte del Departamento de Ciencias de la Educación de la UCH CEU, de entre cuyos miembros fueron dos profesoras ${ }^{2}$ las encargadas del proyecto. Para la movilidad, aparte de las docentes mencionadas, se seleccionaron ocho estudiantes ${ }^{3}$. En una primera fase, se informó a los alumnos y alumnas de los grados de Educación Infantil y Educación Primaria acerca de este proyecto de cooperación. El alumnado interesado debía presentar una carta de motivación, en la que, además de justificar su interés en participar, se debían explicar, en su caso, experiencias previas similares. Finalmente, la selección de las candidaturas la llevó a cabo un comité compuesto por las profesoras participantes y por el coordinador de la Cátedra Solidaridad, teniendo en cuenta las motivaciones, los conocimientos sobre trabajo colaborativo, la formación ad hoc en ayuda humanitaria y cooperación internacional y la existencia de experiencias previas relacionadas.

Respecto de la comunidad educativa senegalesa, estuvieron implicadas más de 300 personas: los estudiantes de los cinco primeros cursos de Primaria superaban los 200, a

$2 \quad$ Nuria Andreu, del centro de Elche, y Rocío López, de la sede de Valencia.

3 Todos ellos pertenecientes a la sede de Elche, cuatro del Grado en Educación Infantil (Carolina Cutillas, Inés Mira, Jorge Llinares y Rocío Priego) y cuatro del Grado en Educación Primaria (Andrea Bolívar, Carmen Berenguer, Cristina Fuentes y Stella Doménech). 
quienes hay que sumar sus profesores ${ }^{4}$. Por su parte, en la escuela infantil de 3 a 5 años había matriculados 90 niños entre los tres cursos, que participaron en el proyecto junto con cuatro maestros ${ }^{5}$. Los centros educativos a los que nos referimos son la Guardería Antoni Gaudí y la Escuela Primaria de Djikesse. Ambos se encuentran en Djikesse, una pequeña aldea de 2253 habitantes, en el departamento de Bignona, perteneciente a la región de Ziguinchor, en Senegal. Se trata de una zona con gran carencia de recursos tecnológicos, algo que se observa no solo en educación (Seye Djité y Diakhate, 2019), sino, especialmente, en las limitaciones para hacer frente a las labores agrícolas, que se limitan al autoconsumo.

Entre los organismos implicados en el proyecto, participa la Asociación GUP, en calidad de partner de la Universidad para la Misión Senegal. Es una organización sin ánimo de lucro adscrita a la Parroquia de Nuestra Señora de la Asunción, del barrio de Benimaclet (Valencia). En 2011, la Asociación GUP comenzó a colaborar en estos proyectos, fundados por Albert Ballerini hace más de veinte años, llegando a tomar el relevo posteriormente, tras su fallecimiento. Ballerini había llegado en 1997 a Senegal para realizar una labor misionera y, a partir de las necesidades de la gente de Djikesse, construyó un jardín de infancia (Guardería Antoni Gaudí) y una huerta cooperativa, con el objetivo de que las familias pudiesen recoger alimentos para ser autosuficientes. La creación de esta guardería, con capacidad para 100 plazas, se llevó a cabo por dos razones de urgencia: la carencia de centros y la alta natalidad en la aldea, siendo además frecuentes los matrimonios y embarazos precoces. Actualmente, también se están encargando de la rehabilitación de la Escuela Primaria de Djikesse y de la provisión de recursos.

\section{Descripción y resultados del proyecto}

\subsection{Planificación y preparación}

El diseño del proyecto, que se presentó a la Cátedra Solidaridad del Vicerrectorado de Estudiantes y Vida Universitaria de la UCH CEU en julio de 2019, planteaba ya entonces la descripción de los objetivos de este, así como la planificación de la intervención educativa que se llevaría a cabo, teniendo en cuenta la organización escolar senegalesa, al menos a nivel formal (Seye Djité y Diakhate, 2019).

Económicamente, se solicitó a la cátedra el importe de los billetes de avión de las docentes que iban a viajar, así como su alojamiento y dietas, dado que los materiales se recogerían de manera solidaria por el resto de la comunidad educativa universitaria. También se previó una ayuda económica para el alumnado, que fue destinada a adquirir «pulseras solidarias» con cuya venta ayudar a sufragar el viaje.

Tras la fase de planificación del proyecto, y una vez aprobado el mismo, tuvo lugar la fase de preparación de la expedición, que se extendió durante el primer cuatrimestre del curso 2019-2020. Esta fase se reveló como de especial importancia, tanto para disponer lo necesario de cara a la intervención como para favorecer el desarrollo de actitudes y compromisos. Durante esta, en las sedes de la universidad a las que pertenecen las

$4 \quad$ Albert Sadio, director del centro y maestro del cours d'initation (CI); Youssouph Diédhiou, maestro del cours préparation (CP); Djibril Mary, maestro del cours élémentaire première année (CE1); Gnansou Camara, maestro del cours élémentaire deuxième année (CE2); y Abba Bayo Sané, maestro del cours moyen première année (CM1).

$5 \quad$ Assi Diallo, maestra de la petite section (PS); Nana Coly, maestra de la moyenne section (MS); Aimé Pascal Manel, director y educador de la grande section (GS); y Mamadou Manga, educador suplente). 
profesoras, se fijaron puntos de recogida de material escolar, así como de materiales para confeccionar recursos didácticos, que se llevaría a la Guardería Antoni Gaudí y a la Escuela Primaria de Djikesse, tanto para el desarrollo del proyecto como para dotar de recursos a los centros una temporada. Esta llamada, a la que podía responder cualquier estudiante, profesor o profesora de la Facultad, o de la Universidad en general, se difundió especialmente entre el alumnado de Magisterio, convirtiendo así esta misión educativa en «algo de todos», un esfuerzo que fue recogido en prensa (Actualidad CEU, 2020b; Información, 2020b) y que se tradujo en más de $300 \mathrm{~kg}$ de material entre mochilas, cuentos, reglas, tijeras, lápices, rotuladores, ceras, pinturas y pinceles, gomas de borrar, bolígrafos, libretas, folios, saltadores, flautas, plastilina, pegamento...; y también materiales para confeccionar recursos didácticos, como cartulinas, corcho, papel pinocho, chinchetas, anillas, velcro, tapones, goma eva, fieltro, pajitas, pinzas de la ropa, lanas...

La planificación tenía en cuenta la explotación pedagógica de estos materiales, por lo que estaba previsto explicar y mostrar, in situ, para qué podían servir, cómo utilizarlos en el aula y con qué objetivos didácticos, es decir, qué actividades se habían pensado para usarlos, siendo conscientes de que las posibilidades son múltiples y ellos mismos podrían desarrollar otras propuestas. De entre las posibilidades, algunas fueron programadas de manera completa con los estudiantes de Magisterio, en la fase de preparación del proyecto, para llevarlas a cabo en las escuelas senegalesas, incluyendo la confección de materiales didácticos.

Las características de los materiales que se diseñaron y elaboraron primaron la facilidad en el proceso y la versatilidad de uso: eran sencillos, manipulativos y susceptibles de servir para utilidades muy diversas. La idea es que permitieran trabajar cálculo, psicomotricidad, valores y actitudes...

\subsection{Intervención y descripción del contexto educativo senegalés}

El período de ejecución del proyecto tuvo lugar a comienzos del segundo semestre del curso 2019-2020. Aparte de describir la intervención, cabe comprender las características educativas del país de acogida, centrándolas en las dos escuelas donde se incardinó el proyecto.

\subsubsection{Guardería Antoni Gaudí}

\subsubsection{Espacios}

Dentro del recinto de la escuela infantil existen diferentes zonas, que describimos a continuación. Las aulas de 3 y 4 años son construcciones de forma cilíndrica, con tejado en forma de cono. Son de dimensión reducida, por lo que solo caben dos piezas de mobiliario: la mesa para el profesorado y una estantería pequeña, aparte de la pizarra. Así pues, el alumnado realiza las tareas en el suelo sobre unas alfombras. Para las explicaciones con soporte visual y los ejercicios de los alumnos, las aulas poseen pizarras, dispuestas a una altura óptima para el alumnado. El aula de 5 años es más amplia y luminosa: no necesita iluminación artificial. Dispone del mismo mobiliario que las anteriores, pero, además, rodeando el espacio el aula por dentro, hay una especie de banco corrido de construcción donde, además de servir para acomodar los juegos y materiales con los que se trabaja en clase, en algunas ocasiones se utiliza como lugar de apoyo donde realizar las tareas. 
Con las mismas dimensiones y estructura que las aulas de 3 y 4 años, se encuentra el aula de dibujo, que sí dispone de cinco mesas.

Entre las aulas de 5 y 3 años se encuentran las letrinas, de uso diferenciado por sexos.

El último espacio de uso infantil es el patio, que ocupa todo el entorno que rodea las aulas. Es de tierra, con grandes árboles, y cuenta con bancos y con un tobogán, que se usan durante el recreo y también para realizar las actividades educativas de psicomotricidad gruesa.

El principal espacio de uso exclusivo del profesorado es la estancia de descanso y de curas, que dispone de un baño para adultos, y también de una camilla y un botiquín.

Por su parte, el despacho y almacén se dedica al almacenaje del material que llega a este jardín de infancia, procedente de ONG, aunque también cuenta con un escritorio donde poder trabajar.

La cocina principal se encuentra al aire libre, aunque hay otra supletoria con una nevera para bebida y donde preparar almuerzos. El área es amplia y en ella hay un pozo y un lugar donde es posible hacer focos de fuego para cocinar en grandes ollas, si bien se encuentran en desuso por falta de recursos económicos.

\subsubsection{Aspectos pedagógicos y organizativos del centro}

El horario del centro es de lunes a viernes de 8:30 a 13:00 h, si bien la hora de entrada es orientativa, más flexible de lo que cabría esperar, sujeta a los ritmos de cada familia e, incluso, a la llegada del maestro o maestra. Asimismo, cabe mencionar, dada la prioridad que las familias otorgan a otras necesidades, que hay grandes tasas de absentismo. Ello se alimenta del hecho de que la escolarización no es obligatoria en estas edades, pero aun así hay un gran número de alumnado matriculado: son tres grupos de 3 , 4 y 5 años, de 28, 30 y 32 estudiantes, respectivamente.

$\mathrm{Al}$ respecto, se observan prácticas de agrupamientos adicionales o alternativo: en el aula de 5 años, y a veces en la de 4, cuando el maestro lleva a cabo explicaciones en la pizarra, dada la gran cantidad de alumnado, divide el aula en dos grupos, que realizan la misma actividad, pero en diferentes tiempos: el que está en la parte delantera del aula, donde se sitúa la pizarra, atiende en primer lugar las explicaciones del profesor, y el otro permanece en la mitad posterior, entreteniéndose con juegos, en silencio, a la espera. Luego se intercambian los espacios y actividad. Respecto al aula de 3 años, se suele dividir en tres grupos de trabajo. A cada uno de estos subgrupos se les asigna el nombre de una fruta, siendo este el distintivo a la hora de dirigirse a cada grupo para realizar una actividad.

\subsubsection{Programación curricular, atención a la diversidad y estrategias motivacionales}

Podemos destacar dos fuentes de motivación: por un lado, el propio profesor o profesora, que se encarga de involucrar y guiar al alumnado en su proceso de aprendizaje. Por otro, la motivación entre iguales, por ejemplo, cuando en psicomotricidad se anima a los compañeros dando palmas y proclamando su nombre.

En cuanto al plan de acción educativa para el alumnado, no es en absoluto como lo concebimos en España. Es decir, no existe un currículo programado en bloques de contenido, competencias, actividades, etc. Lo que se hace en esta escuela infantil es plantear semanalmente los contenidos, que quedan expuestos en el tablón del aula, para facilitar su consulta. Esto se observa especialmente en el grupo de 5 años, pues los otros 
presentan menos organización. El esquema de la jornada, no obstante, es siempre el mismo, para facilitar el establecimiento de rutinas: se comienza recitando una poesía, aprendida previamente; se trabaja la psicomotricidad en el patio; se regresa al aula para abordar los contenidos planificados; el resto de la jornada se divide en sesiones cortas de duración flexible (generalmente de una media hora).

Por otro lado, es escasa la acción educativa para dar respuesta específica a las diferentes situaciones culturales, económicas y sociales, a las diferentes motivaciones e intereses, a los diferentes ritmos y estilos de aprendizaje... No hay, pues, atención a la diversidad: los grupos-clase están compuestos por un excesivo número de educandos en relación con los pocos recursos materiales y personales de que disponen, por lo que el trabajo personal, individualizado, se convierte en una tarea imposible de abordar, para la cual tampoco se cuenta con formación previa.

Finalmente, en lo que respecta a la educación especial (acción educativa para dar respuesta a las diferentes capacidades), resulta inevitable señalar el caso de una alumna con dificultades motoras, que se desplazaba en silla de ruedas, si bien lo cierto es que los recursos, tanto humanos como materiales, para el apoyo ante sus necesidades educativas en la realización de las actividades son inexistentes, por lo que podríamos hablar de integración, pero no de inclusión, dado que muchas veces quedaba desatendida y apartada de las actividades.

\subsubsection{Recursos personales y materiales}

En cuestión de recursos, tanto personales como materiales, el centro educativo se encuentra muy limitado. Los primeros podrían ganar en calidad si se atendiese más o mejor a la formación docente. Y, en cuanto a cantidad, las necesidades son evidentes tanto en lo relativo a la atención de la alumna con discapacidad motora como en el hecho de que, cuando un profesor o profesora falta un día a clase, no se le sustituye, sino que se agrupa a los alumnos en alguna otra clase.

En cuanto a los materiales, su escasez fue suplida puntualmente gracias a la aportación del proyecto, que asimismo consistía en enseñarles a elaborar materiales didácticos con recursos habituales o naturales del entono: tapones, arena, hojas..., siempre orientados a favorecer aprendizajes significativos.

Finalmente, cabe tener en cuenta que la participación de las familias en el proceso de enseñanza-aprendizaje es nula, así como su colaboración en otras actividades del centro educativo, que se limita a la limpieza de la escuela por parte de las madres del alumnado. Durante la ejecución del proyecto se pretendía, en este punto, trasladar al equipo docente la importancia de esta participación para que los aprendizajes sean más significativos, que puede empezar por iniciativas sencillas, como la exposición de los trabajos realizados por el alumnado para que las familias pudieran ser partícipes del aprendizaje de sus hijos y esto sirviera de motivación para ellos. Además, teniendo en cuenta la escasa alfabetización de las madres, esta implicación sería beneficiosa también para ellas.

\subsubsection{Acciones desarrolladas en la Guardería Antoni Gaudí}

Como se ha adelantado, durante los meses previos al viaje, tanto desde el grupo del Grado en Educación Infantil como el del Grado en Educación Primaria, se elaboraron materiales didácticos, pensando siempre en que estos fuesen resistentes y duraderos y, también, que pudiesen ser sustituidos por otros recursos materiales (habituales o naturales) de aquel entorno. 
Las actividades programas fueron diseñadas respetando el nivel madurativo de cada edad. No obstante, sufrieron modificaciones teniendo en cuenta el contexto educativo concreto, así como de los conocimientos previos del alumnado. Así pues, a partir de lo observado el primer día, se prepararon actividades nuevas adaptadas a las necesidades.

En todas las actividades se trabajó con metodologías basadas en la experimentación y en la observación, así como promotoras de un aprendizaje globalizador y significativo para el alumnado, además de priorizar la importancia del juego para conseguir un óptimo aprendizaje.

Asimismo, la intervención en la Guardería Antoni Gaudí incluyó la adaptación de espacios, que incluía reagrupamientos y redistribución de objetos. Por su susceptibilidad de replicación, estas dos cuestiones las desarrollamos en el apartado siguiente.

Finalmente, en lo que respecta a los recreos, se trabajó en aumentar su dinamismo y su potencial pedagógico, con juegos, por un lado, así como con muestra de cariño y prestando atención más individual a cada estudiante.

\subsubsection{Escuela Primaria de Djikesse}

\subsubsection{Espacios}

El colegio de Educación Primaria está compuesto por dos aulas de cañizo, para los dos primeros cursos, y otras tres, de cemento. Esta escuela comenzó también como un proyecto de Albert Ballerini, humilde pero ambicioso, al que se sumó el Ministerio de Educación de Senegal colaborando con la Asociación GUP en la renovación de las infraestructuras.

Además, hay una nueva construcción destinada a nuevas aulas, para mejorar las de $1 .^{\circ}$ y $2 .^{\circ}$ y para añadir un $6 .^{\circ}$ curso, así como una estructura con cuatro letrinas y un pozo. Todo lo demás es una gran extensión de tierra que hace las veces de patio, con neumáticos para jugar.

\subsubsection{Aspectos pedagógicos y organizativos del centro}

$\mathrm{Al}$ igual que en España, la Educación Primaria es carácter obligatorio y comienza a los 6 años, hasta los 12. A pesar de ello, en la Escuela Primaria de Djikesse hay únicamente de $1 .^{\circ}$ a $5 \cdot^{\circ}$, con un aula por curso, que acogen a más de cuarenta alumnos cada una. En este caso, el porcentaje de abandono es mínimo, gracias a la implicación de la comunidad y a la motivación del profesorado, cuyo objetivo principal es que estos niños continúen sus estudios.

El horario del centro es de lunes a viernes de 8:00 a 13:00 h. De 11:00 a 11:30 h está establecido un descanso, aunque se suele alargar. De manera complementaria, los martes y jueves por la tarde se atiende al alumnado que va más atrasado, a modo de refuerzo.

La metodología utilizada podría calificarse de tradicional, en tanto que se siguen las ideas, normas y costumbres propias de lo que se veía en España hace medio siglo: una figura docente autoritaria, un papel discente pasivo, un proceso de enseñanza -que no enseñanza-aprendizaje- basado en la repetición, etc.

\subsubsection{Programación, atención a la diversidad y estrategias motivacionales}

El plan de acción educativa tampoco es como lo concibe desde hace años la didáctica, es decir, no con un currículo programado en bloques de contenido, competencias, actividades, etc. Tampoco se ponen en marcha estrategias motivacionales: el alumnado tiene un papel pasivo, basado en el trabajo individual, y la metodología didáctica es monótona, 
basada en la repetición. Por todo ello, el proyecto hizo hincapié en este aspecto a la hora de explicar al equipo docente las ventajas de las metodologías activas, el trabajo cooperativo, en grupo, y el aprendizaje significativo. Estas iniciativas llegaron para quedarse, tal y como ha informado el director del centro con posterioridad a la estancia.

Asimismo, y al igual que en la escuela infantil, se resaltó la importancia de la exposición de las producciones en las paredes del aula, con el objetivo de motivar al alumnado y reforzar su trabajo, además de que también servía para que las familias fueran testigos del aprendizaje.

Tampoco se atendía de ningún modo las diferentes naturalezas y ritmos de aprendizaje del alumnado, dados el excesivo número de educandos, la escasez de recursos materiales y personales, así como la ignorancia de los procedimientos y beneficios individuales y colectivos de la atención a la diversidad. En lo que respecta a la educación especial, puede señalarse el caso de una alumna con síndrome de Down, «integrada» en el grupo de $1 .^{\circ}$ : se sentaba en primera fila, pero carecía de los recursos de apoyo y adaptación deseables. Sentados al final, por el contrario, se encontraban tres alumnos con retrasos no diagnosticados, para los que no existía ningún tipo de consideración específica. Finalmente, en la clase de $2 .^{\circ}$ había un alumno con sordera profunda, para el que tampoco había articulado ningún tipo de adaptación o ayuda. Por todo ello, podemos hablar de integración, pero en ningún caso de inclusión social y educativa, quedando patente que el profesorado no poseía ni la actitud ni la formación necesarias para atender a la diversidad en el aula.

Durante la estancia, en las intervenciones relacionadas con el proyecto sí se tuvieron en cuenta estas circunstancias personales y, al distribuir al personal de dos en dos por las clases, se pudo atender individualmente a estos alumnos durante la realización de las actividades. En este punto se registró un cambio evidente en ellos, pues llamó la atención lo receptivos y motivados que se mostraron.

\subsubsection{Recursos personales y materiales}

Los recursos personales disponibles son muy ajustados, pues se limitan a un maestro para cada grupo-aula, quien imparte todas las materias, y en la misma aula: matemáticas, lengua (francés), ciencias sociales y ciencias naturales. Las ausencias de profesorado tampoco son sustituidas, sino que los alumnos se agrupan en alguna otra clase, se quedan solos con supervisión intermitente o se marchan antes.

El material escolar básico, de uso individual recurrente, consiste en una pizarrita que utilizaban a modo de libreta, en que la que resolvían los ejercicios que el profesor planteaba en la pizarra del aula. Cuando terminaban de resolver la tarea la levantaban para que el profesor la viera; entonces, se acercaban a la parte delantera del aula, donde había un cubo con agua jabonosa y una esponja y la limpiaban.

En el aula disponían de un armario donde guardaban material didáctico procedente de donaciones anteriores, pero este era muy escaso.

Como en la guardería, tampoco en la escuela de Primaria se registra participación de las familias. La única colaboración regular es que algunas madres preparan almuerzos para venderlos en el recreo del colegio a los alumnos a un mínimo precio. Puntualmente, se atiende a las madres o padres en tutoría: en ellas, el profesorado les informa de la evolución académica de su hijo o hija y, eventualmente, se aprovecha para tratar algún tema organizativo o de papeleo. 


\subsubsection{Acciones desarrolladas en la Escuela Primaria de Djikesse}

La metodología planteada para Educación Primaria fue una metodología activa, basada en la experimentación, promoviendo siempre un aprendizaje significativo y también cooperativo. No obstante, su implementación se vio dificultada porque las clases eran muy numerosas y el mobiliario poco ajustable, así como por el desconocimiento de este tipo de planteamientos por parte del profesorado autóctono y, sobre todo, del alumnado: al trabajar en grupo o por parejas, llamó la atención las escasas habilidades sociales para el trabajo colaborativo que mostraron.

El recurso metodológico que sí funcionó desde el principio fue el aprendizaje mediante el juego, que se aplicó, por ejemplo, tanto a la educación física como a ejercicios de matemáticas, mediante juegos tradicionales.

Respecto a la adaptación de espacios, la gran cantidad de alumnos por aula reducía la posibilidad de adaptar espacios y crear rincones, pero sí se pudo dar un toque personal a las clases, decorándolas con pósteres, murales y trabajos realizados por el alumnado. Esto ayuda a que la clase se convierta en un espacio propio y a que se genere un ambiente acogedor.

\subsection{Repercusión de la experiencia de aprendizaje servicio en Senegal}

Como todo proyecto de cooperación solidario, sobre todo desde la metodología del ApS, este también tiene dos beneficiarios. Por un lado, los futuros docentes, estudiantes de la UCH CEU, además de las dos profesoras que los acompañaron e impulsaron el proyecto. Todos ellos volvieron más concienciados con el valor fundamental de la educación como instrumento para combatir la pobreza y la exclusión social (Actualidad CEU, 2020c). Por otro lado, y principalmente, los beneficiarios destinatarios de este proyecto fueron los maestros de las escuelas de Djikesse y, consecuentemente, su alumnado. El objetivo principal sobre el terreno era implementar metodologías activas y enseñar a los docentes a utilizar materiales didácticos, incluso a confeccionarlos, optimizando los procesos de enseñanza-aprendizaje. Y así fue.

\subsubsection{Resultados del servicio en las comunidades educativas senegalesas}

El último día de estancia en las escuelas, una vez finalizadas las clases, se celebraron dos claustros en sendas escuelas con motivo de la despedida del grupo visitante, donde este recibió retroalimentación por parte de cada equipo docente: aseguraron que para ellos habían sido muy ventajosas la intervención y las acciones desarrolladas, que les habían abierto nuevos horizontes de actuación educativa, los cuales, asimismo, compartirían con docentes de otros centros educativos.

Previamente, mediante la observación participante se había recabado más información, que ratificaba y complementaba lo aportado en las reuniones mencionadas y que pasamos a resumir, seleccionado las repercusiones más significativas.

Con todas las acciones emprendidas, se pudo incidir en algunas cuestiones muy concretas de aquella realidad educativa. En la Guardería Antoni Gaudí puede destacarse la promoción de metodologías activas de aprendizaje, haciendo consciente al profesorado de la importancia de la experimentación y la observación, así como de promover siempre un aprendizaje globalizador y significativo para el alumnado. Además, se consiguió resaltar el papel del juego para conseguir un óptimo aprendizaje.

Respecto a los procesos cooperativos, aunque implicaban una metodología considerada importante y prevista para este proyecto, la falta de espacio y de recursos hizo 
necesario aplicarlo con modificaciones, por lo que no se llegó a experimentar su esencia. Aun así, antes del proyecto todas las actividades eran de abordaje individual y los alumnos nunca interactuaban unos con otros. Por ello, aun con las limitaciones comentadas, las actividades propuestas, en su mayoría realizadas en equipo, los iniciaron en la adopción de roles y en la resolución colaborativa.

Otra cuestión en la que hubo una repercusión positiva fue en la organización de espacios y materiales. Se observó que las clases precisaban de un arreglo para un mejor aprovechamiento del espacio, así como resituar los objetos (juguetes, material...) y distribuirlos según su uso. Con ayuda de sus profesoras, el alumnado universitario tomó la iniciativa de agrupar los recursos por secciones diferenciadas. Delimitaron rincones con sus respectivos materiales y carteles, que indicaban qué tipo de materiales se podía encontrar en cada uno de estos espacios. De este modo, el maestro o maestra puede distribuir los materiales con mayor agilidad. Al mismo tiempo, se insistió en la responsabilidad que hay que trasladar a los niños y niñas de dejarlo todo colocado como lo encontraron antes de utilizarlo, evitando así el desorden y el extravío de juguetes y material, cuestión esencial para optimizar los recursos. También, se organizó y puso más al alcance del profesor el material escolar, ordenando en botellas de plástico cortadas por la mitad, a modo de portalápices, colores, tijeras, pegamentos, bolígrafos y muchos otros materiales, para que fuera visible y se pudieran utilizar según conviniese para cada actividad.

Asimismo, se crearon ambientes o rincones de trabajo, como el rincón de la construcción (piezas de madera y de plástico), el de la psicomotricidad (los abalorios), el de escritura y grafomotricidad y el rincón de la lectura, entre otros. Quedó así establecida la posibilidad del trabajo por rincones, cuyas ventajas para Educación Infantil fueron expuestas y debatidas con el profesorado de la guardería.

Para personalizar las clases, estas fueron decoradas con pósteres, frases, carteles y manualidades realizadas por los niños y niñas. Esto ayuda a que la clase se convierta en un espacio «propio» y a que se genere un ambiente acogedor. Precisamente este fue un aspecto que quedó instalado tanto en Infantil como en Primaria, el de dar visibilidad al trabajo que realizaba el alumnado: la importancia de que este pudiera ser mostrado al resto de personas que componen la comunidad educativa, incluyendo a las familias.

Respecto a la repercusión del proyecto en la Escuela Primaria de Djikesse, también el contacto con las metodologías cooperativas fue incipiente, dado que les resultaba bastante desconocido. Por ello, se insistió en explicar a los docentes senegaleses algunos de sus beneficios, destacando que el objetivo principal de trabajar de manera cooperativa es el de desarrollar valores como el respeto, el compañerismo, la colaboración... Una «línea pedagógica que promulga el aprendizaje activo, la autonomía del individuo y la importancia de tomar la palabra; que entiende que hay que educar personas críticas y capaces para construir un mundo más libre y justo» (Mínguez-López et al., 2017, p. 106).

Interesante, asimismo, fue el cambio de mentalidad acerca de la concepción del juego, aspecto totalmente desterrado de las aulas de Primaria dado que, como comentó el director, consideraban estas actividades propias de la etapa Infantil. Esto era porque no trascendían el mero juego o manualidad y las concebían con un fin exclusivamente lúdico. Al explicar los objetivos pedagógicos que perseguían ciertas actividades, fueron conscientes de su potencial didáctico, además de constatar el aumento de motivación discente que se producía durante la ejecución de estas. Relacionado con esto, cabe destacar que, durante la estancia, el equipo visitante hizo mucho uso de un espacio cuyo valor pedagógico estaba abandonado: el patio. Además, se impulsó su valor lúdico organizando 
el tiempo de receso con actividades semidirigidas, de participación voluntaria: se jugaba con los alumnos, se saltaba con ellos a la comba, se cantaba y se bailaba conjuntamente...

Una intervención muy concreta, que repercutió de manera significativa entre los maestros y maestras de Djikesse, fue la de mostrarles cómo captar la atención del alumnado de una manera amable, algo tan sencillo como levantar la mano en silencio y, así, cada estudiante que la va viendo alzada, lo hace igualmente «por contagio». Mediante esta imitación se va alcanzando el silencio en clase y el profesorado logra reconducir la atención del grupo.

Por último, tanto las intervenciones en educación emocional como la de promoción de la convivencia tuvieron un impacto reseñable. Esta última, que hicimos coincidir con el Día Escolar de la No Violencia y la Paz, quedó vinculada a la conmemoración de ese día, fecha que quedó señalada en su calendario académico para futuros cursos y que no pasarán por alto, como había sucedido hasta el momento.

\subsubsection{Resultados del aprendizaje en el alumnado de Magisterio}

Las diferentes dimensiones del proyecto fueron autoevaluadas por los mismos participantes en la iniciativa de aprendizaje servicio, siguiendo la rúbrica expuesta en el apartado de metodología. Los resultados se resumen en la tabla $3(n=10$; es decir, corresponde al $100 \%$ de la población a la que representa).

Tabla 3 .

Resultados de la autoevaluación de proyecto de aprendizaje servicio en Senegal

\begin{tabular}{llllllll}
\hline & I & II & III & IV & MEDIA & Estudiantes & Profesoras \\
\hline (1) B. Necesidades & O & 1 & 1 & 8 & 3.7 & 3.75 & 3.5 \\
\hline (2) B. Servicio & 1 & 3 & 2 & 4 & 2.9 & 3 & 2.5 \\
\hline (3) B. Sentido del servicio & O & 2 & 3 & 5 & 3.3 & 3.125 & 4 \\
\hline (4) B. Aprendizaje & 0 & 1 & 6 & 3 & 3.2 & 3.25 & 3 \\
\hline (5) B. Participación & 0 & 1 & 6 & 3 & 3.2 & 3.125 & 3.5 \\
\hline (6) P. Trabajo de grupo & 0 & 1 & 6 & 3 & 3.2 & 3.125 & 3.5 \\
\hline (7) P. Reflexión & 0 & 2 & 4 & 4 & 3.2 & 3.125 & 3.5 \\
\hline (8) P. Reconocimiento & 1 & 2 & 3 & 4 & 3 & 2.875 & 3.5 \\
\hline (9) P. Evaluación & 3 & 1 & 2 & 4 & 2.7 & 3.125 & 1 \\
\hline (10) O. Partenariado & 1 & 0 & 6 & 3 & 3.1 & 3 & 3.5 \\
\hline (11) O. Consolidación centros & 3 & 2 & 2 & 3 & 2.5 & 2.125 & 4 \\
\hline (12) O. Consolidación entidades & 2 & 3 & 2 & 3 & 2.6 & 2.5 & 3 \\
\hline
\end{tabular}

Fuente: elaboración propia a partir de la rúbrica de Serrano et al. (2015, pp. 120-121).

Como se observa en las casillas señaladas, todos los ítems obtuvieron mayor número de respuestas en las puntuaciones III y IV, con la excepción de los dos últimos, que presentan una distribución curiosa. Estos, junto con la categoría 9, fueron los peor puntuados de media, a los que sigue el ítem 2. Si desglosamos las medias según el rol de los participantes, observamos un fenómeno llamativo: los dos ítems peor valorados por las docentes ( 2 y 9) no son considerados tan negativamente por parte del alumnado. Por el contrario, los ítems más bajos ( 11 y 12) superan el corte III para las docentes, llegando a IV precisamente el ítem 11. Finalmente, los discentes valoran con menos de III un tercer ítem, el 8, si bien la media y las medianas lo mantienen por encima. 
Si analizamos cualitativamente estos ítems, vemos que, dentro de los básicos, el 2 (servicio) es valorado como «II. Continuado» («Servicio de duración prolongada compuesto por tareas repetitivas y/o fáciles de aprender, cuya realización supone una exigencia e implicación moderadas»), pero, sobre todo, como «IV. Creativo» («Servicio de duración variable compuesto por tareas complejas que los propios participantes deben diseñar para resolver un problema que exige creatividad, lo que supone incluso una exigencia e implicación mayores»). Así pues, para mejorar estos resultados, la implicación del alumnado en la fase de planificación y preparación se revela como muy necesaria para este aspecto del proyecto.

La categoría 9 (evaluación) es valorada con calificaciones aún más extremas: aunque cuatro personas le asignan IV («Conjunta. Los participantes, junto con los educadores, intervienen de manera activa en diferentes momentos del proceso de preparación y aplicación de un plan de evaluación competencial»), cabe decir que por I («Informal. No existe un plan de evaluación establecido, aunque los educadores, de manera espontánea y puntual, pueden evaluar y comunicar su valoración a los participantes») se deciden otras tres, dos de ellas las profesoras, lo que hace pensar que el alumnado no era consciente de que no había un plan evaluativo establecido o, quizás, pensó que la evaluación espontánea que se dio era lo establecido.

También la categoría 11, relativa a la consolidación de centros, repartió sus resultados, principalmente, entre I («Incipiente. El aprendizaje servicio se conoce a causa de un proyecto que ya lleva a cabo algún educador/a o debido a la presentación de una experiencia de otro centro») y IV («Identitaria. El aprendizaje servicio forma parte de la cultura del centro, consta en su proyecto educativo y el centro lo presenta como un rasgo de su identidad»). Cabe destacar, sin embargo, que en este aspecto organizativo las docentes son unánimes en valorarlo como IV, quizás porque tienen una mayor conciencia de las iniciativas de la universidad.

Por su parte, la consolidación de entidades (12) se valoró tanto con «II. Aceptada» ( «Los proyectos de aprendizaje servicio tienen el reconocimiento de la dirección y de los profesionales de la entidad, que aceptan la función educadora de la organización») como con «IV. Identitaria» («El aprendizaje servicio forma parte del ideario de la entidad, que lo presenta como un rasgo propio de su identidad y lo dota de los recursos necesarios para asegurar su implementación»), si bien las docentes no se decantaron ni por una ni por otra, sino que, conscientes de sus avances y limitaciones, identificaron esta categoría como «III. Integrada» («El aprendizaje servicio está presente en el programa de actividades de la entidad, que cuenta con la estructura y el personal necesarios para asegurar su implementación»).

La dispersión de las puntuaciones en las respuestas de los estudiantes a estos dos últimos ítems hace pensar que quizás no fueron bien comprendidos, por lo que cabe interpretar los resultados de manera cautelosa.

De entre los ítems valorados positivamente, destaca significativamente el primero, relativo a las necesidades de la comunidad destinataria. Estas son valoradas como «III. Decididas» («Los participantes, a menudo junto con los educadores, deciden las necesidades sobre las que quieren actuar, a través del análisis de diferentes problemáticas y la elección de una de ellas») y, sobre todo, como «IV. Descubiertas» («Los participantes descubren las necesidades al realizar un proyecto colectivo de investigación en el que llevan a cabo un trabajo de comprensión crítica de la realidad»). 


\section{Discusión y conclusiones}

Los resultados del proyecto, al haber sido planteado desde la metodología del ApS, evidencian diversos beneficios tanto para la comunidad educativa visitante como para la de acogida: esta recibió el servicio con gratitud y reconoció el interés por continuar la iniciativa, y aquella volvió concienciada del valor fundamental de la educación como instrumento para combatir la pobreza y la exclusión social. Al igual que demuestran los estudios de López Herrerías (2010) o Rodríguez Gallego (2014), esta experiencia de aprendizaje servicio ha conseguido vincular la teoría con la práctica, además de mostrarse como una metodología que fomenta el desarrollo comunitario, la cohesión de las comunidades y el compromiso responsable. La discusión de los resultados puede complementarse con la alusión a los datos que recientemente han analizado García-Gutiérrez y Corrales Gaitero (2021), quienes refuerzan la idea de que, como institución formativa e investigadora, dentro de las misiones de la Universidad está la cívica o social. Según los resultados de su estudio, esta «tercera misión» ha encontrado en la metodología del ApS un enfoque apropiado para su cumplimiento.

Si nos centramos en recopilar, resumidamente, la parte de servicio, la repercusión en las comunidades educativas senegalesas puede agruparse en cuatro categorías, tanto respecto de la Escuela Primaria de Djikesse como de la Guardería Antoni Gaudí: el avance en metodologías activas y cooperativas, fomentando el valor del juego como recurso de aprendizaje; la aportación de material y pautas para su elaboración, junto con el desarrollo de actividades basadas en ellos, que priorizaran la experimentación y la observación; la adaptación y optimización de espacios, tanto de las aulas como de los patios de las escuelas. A un nivel más pedagógico, relacionado con los procesos de enseñanza-aprendizaje, aparte de la necesidad de pasar al trabajo grupal, en favor del desarrollo de habilidades sociales y comunicativas, se destacaron las ventajas de usar materiales didácticos manipulativos, que favorecen los aprendizajes y estimulan la motivación; también de atender a la diversidad, a los diferentes ritmos de aprendizaje, a los alumnos con dificultades, así como de recurrir al refuerzo para establecer e incrementar conductas positivas y aprendizajes.

Los equipos docentes senegaleses de la escuela infantil y la escuela primaria, en su conjunto mediante las reuniones formales, así como sus miembros de manera individual y espontánea en diversas ocasiones, se mostraron muy agradecidos y motivados con la gran cantidad de material entregado, las actividades mostradas y las metodologías implementadas.

En cuanto a la evaluación del proyecto desde el punto de vista del aprendizaje autoevaluación-, los resultados de la rúbrica utilizada muestran puntuaciones de III y IV en casi todos los ítems considerados. De entre los peor valorados, se concluye que es necesaria una mayor implicación de los estudiantes en la fase de planificación y preparación del proyecto (ítem 2), especialmente en lo relativo a la evaluación de este (ítem 9). Estos son los dos puntos más débiles del proyecto.

Finalmente, como punto fuerte, los propios participantes reconocen como beneficiosa su participación en la toma de decisiones acerca de las necesidades sobre las que actuar, y el hecho de ir descubriéndolas, lo que conduce a una comprensión crítica de la realidad (ítem 1 ).

En definitiva, la presente investigación se suma a las precedentes en esta línea para mostrar los beneficios de estas iniciativas de cooperación, que impulsan la puesta en 
práctica de metodologías docentes más dinámicas e innovadoras en estas sociedades emergentes. Es necesario este tipo de acercamiento entre poblaciones tan diferentes para que, con el conocimiento que en unos países se ha tenido la oportunidad de desarrollar, se coopere con los otros y se impulse su transformación.

\section{Referencias}

Actualidad CEU (2020a, 13 de enero). Formando a docentes en Senegal. https://medios. uchceu.es/actualidad-ceu/formando-a-docentes-en-senegal/

Actualidad CEU (2020b, 23 de enero). Más de 300 kilos de material docente para Senegal. https://medios.uchceu.es/actualidad-ceu/mas-de-30o-kilos-de-material-docentepara-senegal/

Actualidad CEU (2020c, 10 de febrero). Cuando las sonrisas de los niños iluminan a los futuros maestros. https://medios.uchceu.es/actualidad-ceu/cuando-las-sonrisasde-los-ninos-iluminan-a-los-futuros-maestros/

AlicanteHoy.es (2020, 13 de enero). Alumnos y profesores de Magisterio del CEU de Elche formarán a docentes en Senegal. http://alicantehoy.es/2020/01/13/ alumnos-profesores-magisterio-del-ceu-elche-formaran-docentes-senegal/

Álvarez Castillo, J. L., Martínez Usarralde, M. J., González González, H., y Buenestado Fernández, M. (2017). El aprendizaje-servicio en la formación del profesorado de las universidades españolas. Revista Española de Pedagogía, 75(267), 199-217. https://doi.org/10.22550/REP75-2-2017-02

Battle, R. (2009). El servicio en el aprendizaje servicio. En J. M. Puig Rovira (coord.), Aprendizaje servicio (ApS): educación y compromiso cívico (pp. 71-91). Graó.

Folgueiras, P., Gezuraga, M., y Aramburuzabala Higuera, P. (2019). Los procesos participativos en aprendizaje-servicio. Bordón. Revista de Pedagogía, 71(3), 115131. https://dialnet.unirioja.es/descarga/articulo/7163599.pdf

García-Gutiérrez, J., y Corrales Gaitero, C. (2021). Supranacional higher education politics on the "third mission" of University: the case of service-learning. Revista Española de Educación Comparada, 37, 256-280. https://doi.org/10.5944/ reec.37.2021.27535

Hernández, A. (2019). Movilidad y cooperación internacional en la formación de docentes indígenas interculturales. Revista Internacional de Comunicación y Desarrollo (RICD), 3(10), 59-76. https://dialnet.unirioja.es/descarga/articulo/7155144.pdf

Huarcaya, L., Aranda, R., y Atasi, G. (2005). La intervención de las universidades en los proyectos de cooperación al desarrollo formación del profesorado de educación especial en Cuzco. Tendencias Pedagógicas, 10, 61-106. https://dialnet.unirioja. es/descarga/articulo/1407967.pdf

Información (2020a, 15 de enero). Alumnos y profesores de Magisterio del CEU formarán a docentes en Senegal. P. 28. 
Información (2020b, 22 de enero). Alumnos y profesores del CEU recogen más de 300 kilos de material docente para Senegal. P. 37.

Información(2020c, 12 defebrero). Expedición delCEUaSenegal: Unproyectoeducativo solidario. https://www.diarioinformacion.com/universidad/2020/02/12/ expedicion-ceu-senegal-proyecto-educativo/2233722.html

Información (2020d, 26 de febrero). Estudiantes y profesores del CEU muestran las diferencias educativas entre España y Senegal. P. 35.

JRS (2019). Being with Refugees. Annual Report 2019. https://jrs.net/wp-content/ uploads/2020/o9/JRS-ANNUAL-REPORT-2019_Web.pdf

Lear, D., y Abbott, A. (2019). Aprendizaje-servicio y aprendizaje comunitario. En J. Muñoz Basols, E. Gironzetti y M. Lacorte (coords.), The Routledge Handbook of Spanish Language Teaching: metodologías, contextos y recursos para la enseñanza del español L2 (pp. 477-490). Routledge.

López Herrerías, J. A. (2010). Educación de calidad y en competencias para la competitividad o para la cooperación. Revista Complutense de Educación, 21(1), 107-122.

Martín García, M. J., Rubio i Serrano, L., Batlle, R., y Puig Rovira, J. M. (2010). ¿Qué es aprendizaje servicio? En M. J. Martín García y L. Rubio i Serrano (coords.), Prácticas de ciudadanía: diez experiencias de aprendizaje servicio (pp. 15-24). Octaedro.

Mínguez-López, X., Olmos-Fontestad, N., y Alcantud-Díaz, M. (2017). Cuentos para cambiar la escuela. Un modelo de intervención. En A. S. Jiménez Hernández, J. D. Gutiérrez Sánchez, I. Reis dos Santos, J. J. Leiva Olivencia, C. Silva Sousa, M. I. Iglesias Villarán y D. De Micheli (coords.), Reconstruyendo un mundo con ojos de niñas: entre la pobreza y la educación (106-115). GEU.

Monzó, M., Rifà, J., y Rubio, L. (2018). Implementación y extensión del aprendizajeservicio en las entidades de justicia global y cooperación internacional en la ciudad de Barcelona. RIDAS. Revista Iberoamericana de Aprendizaje Servicio, 6, 127140. https://revistes.ub.edu/index.php/RIDAS/article/view/27288

Onda Cero (2020). Alumnos y profesores de Magisterio del CEU inician un proyecto de cooperación en Senegal. https://www.ondacero.es/emisoras/comunidadvalenciana/elche/directo/alumnos-y-profesores-de-magisterio-del-ceu-inicianun-proyecto-de-cooperacion-en-senegal_202001145e1db1b7ocf28446bobf5643. html

Psifidou, I., y Ranieri, A. (2020). European cooperation in vocational education and training: towards a common ambition. Revista Española de Educación Comparada, 36, 32-53. https://doi.org/10.5944/reec.36.2020.26879

Rodríguez Gallego, M. R. (2014). El Aprendizaje-Servicio como estrategia metodológica en la Universidad. Revista Complutense de Educación, 25(1), 95-113. https://doi. org/10.5209/rev_RCED.2014.v25.n1.41157 
Rubio i Serrano, L. (2009). El aprendizaje en el aprendizaje servicio. En J. M. Puig Rovira (coord.), Aprendizaje servicio (ApS): educación y compromiso cívico (pp. 91-106). Graó.

Serrano, L. R., Puig Rovira, J. M., García, X. M., y Rodríguez, J. P. (2015). Analizar, repensar y mejorar los proyectos: una rúbrica para la autoevaluación de experiencias de aprendizaje servicio. Profesorado. Revista de Currículum y Formación de Profesorado, 19(1), 111-126. http://www.ugr.es/ recfpro/rev191ART6.pdf

Seye Djité, S., y Diakhate, M. (2019). Organización del sistema educativo senegalés. $M L S$ Educational Research (MLSER), 3(1), 79-92. https://doi.org/https://doi. org/10.29314/mlser.v3i1.86

Tapia, M. N. (2008). Aprendizaje y servicio solidario. Ciudad Nueva.

Uruñuela, P. M. (2018). La metodología del aprendizaje-servicio: aprender mejorando el mundo. Narcea Ediciones. 